\title{
Nontuberculous Mycobacteria Measurement
}

National Cancer Institute

\section{Source}

National Cancer Institute. Nontuberculous Mycobacteria Measurement. NCI Thesaurus. Code 147459

The determination of the amount of organisms that are assigned to the Mycobacterium genus but are not tuberculosis or leprosy-causing in a biological sample. 